\section{Le psoriasis : évolution et révolution}

Louis Dubertret
Institut de Recherche sur la Peau, Pavillon Bazin, Hôpital Saint-Louis, 1 , avenue Claude Vellefaux, 75010 Paris, France. louis.dubertret@free.fr anomalies génétiques et les anomalies des voies de signalisation qui aboutissent à une sécrétion excessive de cytokines pro-inflammatoires, mais aussi de mieux comprendre les interactions entre cellules cutanées et cellules inflammatoires. Les progrès thérapeutiques récents, dans le traitement du psoriasis, reposent avant tout sur la mise en place de techniques permettant un meilleur ajustement des outils thérapeutiques aux besoins des patients. De fait, si le psoriasis met rarement la vie en danger, il atteint souvent gravement la qualité de la vie : l'objectif est donc d'élaborer une stratégie thérapeutique qui améliore cette qualité de vie, que seul le patient peut évaluer. Cette stratégie, permettant d'ajuster les possibilités thérapeutiques à chaque patient, repose sur quatre phases successives: le questionnement, qui permet d'évaluer la gravité de la maladie, les explications, qui permettent au malade de comprendre comment il peut agir sur sa maladie, la négociation, qui devient l'acte médical principal, et doit aboutir à une décision thérapeutique partagée. Il s'agit là d'une révolution, au sens propre, dans la relation entre le médecin et le malade: l'édu-

cation du patient se situe au cœur de la démarche thérapeutique. C'est dans ce contexte que sont apparus de nouveaux médicaments, très intéressants sur le plan scientifique et apportant l'espoir d'une efficacité raisonnable, associée à une bonne tolérance. Ces médicaments sont toutefois très coûteux, ouvrant en cela le débat des possibilités et des limites de la solidarité. <

Parmi les centaines de maladies qui font la complexité de la dermatologie, le psoriasis tient une position phare. Affectant $2 \%$ à $3 \%$ de la population mondiale, il associe une inflammation chronique, une prolifération des cellules de l'épiderme et un trouble de sa différenciation. Il se traduit par l'apparition de plaques rouge, prurigineuses ou douloureuses, couvertes de squames où l'épiderme se renouvelle en cinq à sept jours au lieu de 28 jours. Ces plaques sont souvent symétriques, ce qui suggère un rôle pour le système nerveux. On observe dans le psoriasis différentes activations cellulaires, notamment celle des cellules présentatrices d'anti-

Article reçu le 31 octobre 2005, accepté le 5 décembre 2005 
gènes. On a donc voulu faire du psoriasis une maladie auto-immune. Certaines émotions pouvant provoquer l'apparition d'une poussée de psoriasis, on a également voulu faire de cette maladie une maladie psychosomatique. Mais la caractéristique la plus fascinante du psoriasis est sans doute le phénomène de Koebner: on peut provoquer, en peau apparemment saine, l'apparition d'une plaque de psoriasis par une agression mécanique ou chimique, à condition de léser à la fois l'épiderme et le derme. Cela est d'autant plus important que le psoriasis est une maladie spécifiquement humaine et que le phénomène de Koebner est, de ce fait, le seul modèle expérimental de psoriasis.

\section{Une évolution continue dans la compréhension de la physiopathologie de la maladie}

Il n'est pas de méthode d'investigation nouvelle en biologie cutanée qui ne soit pas aussitôt appliquée à la recherche sur le psoriasis. Cette maladie est donc le lieu privilégié d'observation des progrès en dermatologie et de l'effet de mode en recherche. Plus de 300 publications par an, et ce depuis plus de 40 ans, ont créé plus de confusion que de compréhension. Ainsi existe-t-il une confusion fréquente entre les anomalies existant dans la peau non lésée, les événements aboutissant à l'apparition d'une plaque (phénomène de Koebner), les anomalies responsables de la chronicité des lésions et les mécanismes responsables de la limitation ou de la régression des lésions. Depuis 17 ans, I'observation de l'efficacité remarquable de la cyclosporine dans le traitement du psoriasis a entraîné une convergence de tous les efforts vers le rôle possible des lymphocytes $T$, ce qui a même amené à considérer le psoriasis comme une maladie auto-immune, dont il fallait rechercher l'autoantigène. Cet effet de mode est si violent que certains chercheurs n'hésitent pas à proposer un schéma de la physiopathologie du psoriasis semblable à celui de l'eczéma de contact (en remplaçant un antigène exogène par un hypothétique auto-antigène), ces deux maladies n'ont pourtant rien à voir sur le plan clinique ou thérapeutique. Il faut rappeler que le psoriasis est avant tout une réponse anormale aux agressions, il ne peut donc être étudié que par une approche dynamique rendant possible l'étude de la réponse cellulaire à différentes agressions. Malgré ces difficultés conceptuelles résultant d'un dialogue insuffisant entre cliniciens et chercheurs, un certain nombre de travaux ont progressivement éclairé la physiopathologie de cette maladie. La comparaison de la peau normale et de la peau non lésée de psoriasis, après greffe du derme et de l'épi- derme sur la souris nue, permet d'observer une hyperprolifération épidermique de la peau non lésée de psoriasis; cette hyperprolifération n'est pas observée si l'épiderme seul est greffé [1, 2]. Ces travaux ont permis de montrer que la peau non lésée de psoriasis est anormale et que le derme est nécessaire à l'expression de cette anomalie. La nature des cellules dermiques impliquées fait l'objet de recherches très actives : fibroblastes, cellules endothéliales, cellules présentatrices d'antigène résidentes, comme les cellules plasmacytoïdes précurseurs de cellules dendritiques et capables de sécréter de l'interféron [3] ? Les fibroblastes cultivés à partir d'une peau non lésée de psoriasis provoquent une hyperprolifération de kératinocytes normaux [4], ce qui indique que les interactions dermo-épidermiques sont anormales dans le psoriasis, même en peau non lésée.

Après greffe de peau normale et de peau non lésée de psoriasis sur la souris nue, l'injection intradermique de lymphocytes $T$ provenant de plaques psoriasiques est capable de provoquer l'apparition d'une lésion psoriasique (clinique et histologique) en peau non lésée, mais pas en peau normale [5]: l'apparition d'une plaque de psoriasis résulte donc de l'interaction de certaines sous-populations de cellules inflammatoires avec des cellules cutanées anormales. La co-culture de lymphocytes $T$ activés et de fibroblastes provenant de sujets normaux ou de sujet psoriasique provoque la production de cytokines pro-inflammatoires par les fibroblastes. Cette production est beaucoup plus importante dans les fibroblastes psoriasiques que dans les fibroblastes normaux [6], ce qui permet de comprendre le lien entre les anomalies cutanées et l'activation des cellules inflammatoires.

Deux publications récentes permettent de quitter la mode du psoriasis comme maladie auto-immune liée à une anomalie du lymphocyte $T$ [ 7 , 8] : portant sur l'étude de deux modèles animaux très proches du psoriasis humain, et résultant d'anomalies principalement [7] ou uniquement [8] cutanées, ces travaux permettent d'envisager de nouveau le psoriasis comme une maladie cutanée provoquée par une réponse excessive des cellules cutanées et inflammatoires aux agressions.

La destruction sélective, dans une plaque de psoriasis, de la microcirculation du derme superficiel par laser pulsé à colorant est un traitement efficace. S'il n'empêche pas une rechute rapide dans les lésions récentes, il entraîne, en revanche, une disparition prolongée, voire une guérison des lésions anciennes [9], montrant ainsi que des modifications de la microcirculation jouent un rôle important dans la chronicité de la maladie.

Plusieurs faisceaux d'arguments convergents suggèrent que le psoriasis est un syndrome réunissant, sous un phénotype commun, des maladies déclenchées par des mécanismes différents: les psoriasis familiaux sont associés à des localisations génétiques variées; les psoriasis débutant tôt dans la vie sont différents des psoriasis à début tardif; plus de 12 modèles animaux présentent, à la suite de modifications génétiques différentes, une prolifération et une inflammation de l'épiderme proches de celles observées dans le psoriasis. Ainsi, si les anomalies retrouvées en peau de psoriasis non lésée ont toujours été retrouvées, les événements aboutissant à leur activation et à l'activation des cellules inflammatoires sont sans doute très variés. Cela est d'ailleurs fortement suggéré par l'observation clinique: en 
effet, les poussées de psoriasis peuvent être déclenchées par des événements aussi variés qu'une infection, une irritation cutanée, un changement de saison, la prise de certains médicaments ou certains types de stress.

Au total, on peut actuellement considérer le (les) psoriasis comme le résultat de l'interaction entre des cellules cutanées hyperréactives, en termes de libération de cytokines pro-inflammatoires, et des cellules inflammatoires activées par des processus variés. Si la réaction responsable de l'apparition d'une plaque peut être déclenchée par les cellules cutanées ou par les cellules inflammatoires, les deux partenaires sont toujours nécessaires pour permettre l'apparition d'une plaque, et l'altération progressive des cellules endothéliales favorise l'entretien des lésions. De façon intéressante, de nombreuses analogies ont été trouvées entre les événements moléculaires et cellulaires aboutissant à une plaque de psoriasis et ceux impliqués dans la cicatrisation cutanée : la plaque de psoriasis ressemble ainsi à une cicatrisation qui n'en finit pas [10].

\section{Une révolution dans la prise en charge des patients souffrant de psoriasis : la médecine centrée sur le patient}

La réflexion sur la stratégie de prise en charge du psoriasis a contribué au développement d'une révolution médicale concernant toutes les maladies chroniques: la médecine centrée sur le patient. De quoi s'agit-il?

Les extraordinaires progrès de la médecine au $x x^{e}$ siècle ont été rendus possibles grâce au développement d'une démarche scientifique utilisant les méthodes statistiques pour étudier la physiopathologie et le traitement des maladies sur de larges populations. Ces progrès ont rendu urgent de déplacer maintenant le regard de la maladie vers le malade, et de mettre au point les méthodes permettant d'appliquer aux individus, résolument uniques, des connaissances acquises sur des populations.

Dans la plupart des maladies aiguës, c’est le médecin qui sait évaluer la gravité de la maladie. Dans les maladies dont la gravité est le plus souvent liée à l'atteinte de la qualité de la vie, à un handicap, seul le patient peut évaluer la gravité de cette atteinte, et donc celle de sa maladie. L'évaluation de cette gravité est capitale puisque c'est elle qui va autoriser ou non l'utilisation de médicaments puissants, efficaces, mais comportant éventuellement des effets secondaires. Comme toujours en thérapeutique, c'est le rapport bénéfice/risque qu'il faut évaluer, mais seul le patient peut vraiment évaluer, avec l'aide de son médecin, la réalité du bénéfice, puisqu'il s'agit de la qualité de sa vie. On ne peut donc soigner une maladie chronique sans la participation informée et responsable du patient. Cependant, le patient venant consulter en 2006 n'est pas du tout prêt à évaluer la façon dont sa vie est perturbée par sa maladie, ni, vraiment, à jeter un regard critique et constructif sur les traitements qu'on peut lui proposer. II vit sa maladie comme une agression extérieure dont la médecine doit le débarrasser, comme on guérit d'une maladie infectieuse ou d'une appendicite. II va donc falloir, au fil des consultations, dissiper ce malentendu et déve- lopper une technique de prise en charge permettant au patient de s'approprier sa maladie et les traitements disponibles, afin de poser les fondements d'une véritable négociation thérapeutique aboutissant non pas à une ordonnance, mais à un choix fait par le patient aidé par son médecin.

Les quatre étapes de cette prise en charge sont le questionnement, les explications, la négociation et la prescription.

\section{Le questionnement}

La phase de questionnement va permettre de commencer à faire la connaissance du malade dans son environnement social, familial et professionnel, de savoir dans quel contexte médical et psychologique la stratégie thérapeutique va devoir s'inscrire, d'évaluer avec lui la gravité de son psoriasis, sa capacité à se prendre en charge, les relations objectives et subjectives qu'il a avec sa maladie et avec les différents traitements qu'il a déjà essayés et, bien sûr, ce qu'il attend de la présente consultation. Son métier, et surtout les conditions dans lesquelles il l'exerce, peuvent rendre impossibles certains traitements: ainsi, des horaires extrêmes rendent quasi impossibles les traitements locaux, il en est de même des voyages fréquents qui rendent également impossibles la photothérapie; les repas d'affaires fréquents, la diminution de l'activité physique et l'augmentation des responsabilités professionnelles réalisent un trio particulièrement propice au développement du psoriasis. Les questions sur les traitements antérieurs sont particulièrement instructives. Elles portent sur la nature des traitements, mais surtout sur les stratégies utilisées. Cela permet de tester l'observance du patient vis-à-vis de ses différents traitements et de connaître les raisons éventuelles pour lesquelles elle n'a pas été optimale. L'opinion du patient sur ce sujet permettra de mieux comprendre ce qu'il attend des différents traitements possibles, et de connaître les contraintes qu'il est capable d'accepter. Un traitement a pu décevoir parce qu'il a été utilisé avec une mauvaise stratégie ou parce que le patient a arrêté ce traitement dès que les lésions cutanées ont disparu. Au cours de ce questionnement, on évaluera le degré de découragement du malade face à son psoriasis et on commencera à identifier quels types de contraintes thérapeutiques le malade est disposé à assumer. On pourra, alors, aborder une question centrale pour la prise en charge thérapeutique : quelle est la gravité du psoriasis et dans quel contexte survient-il ?

Si ce psoriasis est un nouveau venu, il va falloir gérer éventuellement une situation de crise, et le malade n'imagine pas spontanément être impliqué dans son 
psoriasis. Son modèle thérapeutique est souvent celui de l'antibiotique qui permet la guérison en tuant un envahisseur extérieur, ou celui de la chirurgie qui sépare, à l'aide d'un scalpel, ce qui est bon de ce qui est mauvais. La phase de questionnement va alors être particulièrement importante, car elle va modifier insensiblement, par la nature même des questions posées, le scénario à l'intérieur duquel raisonne le malade. II va découvrir progressivement que son psoriasis exprime peut-être quelque chose et, en tout cas, retentit sur beaucoup des relations qui animent sa vie quotidienne. Il découvre que c'est sa propre image qu'il va falloir gérer avec l'aide de son médecin, que c'est sa qualité de vie qu'il va falloir améliorer, et que, de ce fait, il est essentiel qu'il participe au choix thérapeutique. Le questionnement va alors s'orienter vers le stress, vers les possibles conflits de territoire, vers la qualité des relations que le malade a avec lui-même et avec les autres, vers les éléments essentiels qui définissent pour lui la qualité de sa vie.

Ce psoriasis est-il un vieux compagnon auquel on s'est progressivement adapté, tant bien que mal ? Si c'est le cas, il va falloir évaluer comment ce psoriasis a pu modifier les relations que le malade a avec lui-même, avec ses proches, son conjoint ou ses enfants. Ce psoriasis lui $a-t-i l$ fait manquer des occasions, $a-t-i l$ influencé de façon négative sa carrière professionnelle, sa possibilité de choisir un sport, sa façon de s'habiller, sa possibilité de choisir ses vacances? Pense-t-il que ce psoriasis est apparu à l'occasion d'un événement de vie? Les circonstances qui ont déclenché l'expression de la maladie sont-elles encore d'actualité ? Quels sont les projets du malade pour modifier ces circonstances et prendre ainsi, d'une certaine façon, le contrôle de sa maladie, ou tout au moins de ce qu'exprime sa maladie? Le malade peut-il accepter de voir disparaître son psoriasis pour une période particulière qu'il a choisie, puis de vivre une rechute sans être encore plus découragé qu'auparavant? Peut-il accepter des traitements intermittents? La souffrance psychologique entraînée par le psoriasis est-elle constante, ou plus importante à certains moments de l'année ou du fait de certaines localisations? Cette souffrance est-elle disproportionnée par rapport à l'importance des lésions? On soupçonnera alors que le psoriasis a révélé une fragilité psychologique sous-jacente qu'il faudra prendre en charge pour elle-même, avec l'aide d'un psychologue ou d'un psychiatre.

Dans une maladie chronique, le malade réorganise sa vie en fonction de cette maladie pour en souffrir le moins possible. Il est important d'évaluer cette restructuration pour envisager, avec le malade, la meilleure façon de gérer les diverses possibilités offertes par les médicaments et les nombreuses stratégies possibles de leur utilisation. On pourra ainsi progressivement définir dans quel type de scénario thérapeutique le malade, pris dans sa globalité, peut s'inscrire : faut-il un traitement d'urgence, pour faire disparaître les lésions, et profiter de cette accalmie pour aider le malade à reprendre le contrôle sur ce qui était dérégulé dans sa vie, puis faire porter tout l'effort sur la mise en place d'un traitement d'entretien avec tout l'apprivoisement réciproque que cela suppose entre les trois partenaires que sont le malade, son médecin et les médicaments? Faut-il, au contraire, choisir une stratégie centrée d'emblée sur le long terme, à travers une amélioration très progressive de la qualité de la vie?

Toute cette étape de questionnement ne se termine pas lorsque commencent les explications, bien au contraire. Ces explications sur la maladie et sur les traitements vont permettre de poser de nouvelles questions, de faire comprendre leur importance, d'augmenter la qualité des réponses puisque le malade, à travers toutes les explications données, sera de plus en plus à même de se remettre lui-même en question et donc de choisir plus librement.

\section{Les explications}

Elles doivent être données sur le psoriasis et sur les traitements. Elles ont pour but de permettre au patient de participer au choix thérapeutique. Le psoriasis une maladie génétique, multigénique, révélée et non provoquée par des facteurs d'environnement : maladies infectieuses, changements de saisons, stress, médicaments, irritations cutanées, bref par tout ce qui accélère le renouvellement cutané. Lesquels parmi ces facteurs sont pertinents pour le patient? La plaque de psoriasis est la conséquence d'un renouvellement trop rapide de la peau. Lorsque la desquamation devient visible, une réaction inflammatoire se développe qui agresse la peau et entretient la maladie. Le seul fait de gratter la peau, ou d'arracher quelques squames accélère le renouvellement de la peau : cinq minutes de grattage suffisent à réactiver le psoriasis pendant deux semaines. La lutte contre les démangeaisons est donc un élément capital du traitement du psoriasis. Enfin, on explique que le psoriasis n'est pas contagieux. II ne met pas la vie en danger, sa gravité dépendant de son retentissement sur la qualité de la vie. C'est donc le patient, et lui seul, qui sait si son psoriasis est grave, et donc s'il peut relever ou non de traitements majeurs, nécessitant une surveillance rapprochée et comportant des risques.

Tous les traitements freinent, par des mécanismes variés, le renouvellement de la peau. Si le traitement est arrêté dès que les plaques ont disparu, la rechute est rapide. Il est essentiel donc de continuer à se traiter même lorsque les lésions ont disparu: six mois après la disparition d'une plaque de psoriasis, la peau reste tout à fait anormale au niveau microscopique. C'est pourquoi on continue habituellement le traitement un an après que les lésions aient disparu. On insiste également sur le fait que le but du traitement est d'améliorer la qualité de la vie. C'est pourquoi seul le patient peut réellement en évaluer l'efficacité : il n'est pas condamné à se soigner de façon continue, mais peut le faire seulement à certains moments de l'année, ou ne soigner que certaines localisations en fonction de son équation personnelle 
entre la gêne entraînée par la maladie et celle entrầnée par les traitements.

Ces fondements posés, la seconde partie de la phase des explications porte sur chacun des traitements locaux et généraux disponibles, en expliquant leur technique d'utilisation, les contraintes dans la vie quotidienne, les effets secondaires et la stratégie de la surveillance.

\section{La négociation}

Dans l'optique d'une médecine centrée sur le patient, c'est la phase la plus importante de la prise en charge thérapeutique. Elle évolue de consultation en consultation, au fur et à mesure que, à travers questionnement et explications, le malade est de mieux en mieux informé. Il devient capable de dialoguer d'égal à égal avec son médecin, avec un point de vue bien sûr différent et complémentaire.

L'objectif de la négociation est de trouver le meilleur compromis entre les contraintes liées à la maladie et aux traitements et les bénéfices potentiels de chaque traitement, association thérapeutique et succession de traitements. Comme dans toute bonne négociation, chacun doit en sortir, le médecin et le patient, en ayant le sentiment d'avoir choisi le meilleur compromis possible dans une situation précise et à un moment donné. C'est la condition de l'observance et de l'efficacité thérapeutique.

\section{La prescription}

Ce n'est en aucun cas « une ordonnance », mais un contrat qui conclut une négociation. Idéalement, ce contrat de soin devrait être signé par le médecin et par le malade. Cette nouvelle approche thérapeutique se décline différemment dans les diverses spécialités médicales. Elle a pour objectif, à travers une révolution, au sens propre, de la relation médecin-malade, de rendre au malade sa liberté face à l'emprisonnement relatif que provoque sa maladie et auquel contribue parfois le médecin.

Cette modification du regard, à travers l'application de ces techniques, a permis de prendre conscience de toutes les conséquences de la maladie sur la qualité de vie du patient, d'identifier le handicap peau et l'exclusion sociale qui en résulte, de considérer que la souffrance psychologique est aussi importante à prendre en charge que la souffrance physique, que le but des traitements est non seulement de faire disparaître les symptômes mais, de façon plus globale, d'améliorer la qualité de la vie et, dans le cadre des maladies de la peau, de rendre au patient toute sa liberté dans ses relations avec luimême et avec les autres.

La difficulté majeure à la diffusion de cette technique médicale est qu'elle prend du temps (environ 45 minutes par consultation), alors que la valeur économique du temps médical n'est pas reconnue par les système d'assurance.

\section{Un nouveau regard sur l'évaluation de l'efficacité des médicaments}

Le regard médical du $x x^{e}$ siècle s'est passionné pour la maladie avec l'efficacité que l'on sait, au point souvent d'oublier le malade. Les techniques d'évaluation des médicaments ont suivi une évolution parallèle: soucieuses de rigueur scientifique et d'objectivité, elles ont limité l'évaluation de l'efficacité d'un médicament à la mesure de l'évolution de scores quantitatifs, en développant insuffisamment l'évaluation du service rendu, de l'utilité, plus difficile à appréhender. Dans le psoriasis, c'est tout naturellement l'évolution de la surface des lésions qui a été considérée comme la mesure de l'efficacité thérapeutique.

La première difficulté vient de l'évaluation de la surface atteinte. Elle a d'abord été évaluée par la mesure du PASI (psoriasis area and severity index), qui a eu le mérite de permettre la réalisation d'une multitude d'études thérapeutique avec une méthodologie commune. Elle a deux inconvénients : certains dermatologues essaient de se transformer en analyseur d'images, d'autres considèrent que lorsqu'une zone du corps est atteinte, c'est toute la zone qui, fonctionnellement, est atteinte. Pour améliorer le PASI, deux approches ont été proposées : la mise en place de scores globaux, rustiques mais robustes, à la mode aux ÉtatsUnis. Cependant, il est clair que ces scores globaux sont insuffisants, dans la mesure où la cinétique de disparition des plaques n'est pas la même d'une région du corps à l'autre, et que le retentissement sur la qualité de vie de ces différentes localisations n'est pas le même. Une autre possibilité est, au contraire, de proposer des scores non plus globaux, mais analytiques, n'impliquant plus une mesure de la surface de plaques, mais une évaluation des régions du corps atteintes associée, pour chacune de ces régions, à l'évaluation de l'impact de cette atteinte sur la qualité de vie du patient. Un de ces scores, déjà validé [11] et accepté par l'EMEA (Agence européenne d'évaluation des médicaments), est en cours de revalidation.

Une autre difficulté provient de l'utilisation d'un score. Peut-on comparer deux patients en termes de diminution de score? II peut être extrêmement difficile de réduire de $75 \%$ la surface d'un psoriasis peu étendu, limité aux zones résistantes du corps, et extrêmement facile de réduire de $95 \%$ la surface d'un psoriasis très étendu, atteignant des zones très sensibles aux traitements. Ainsi, plus le score initial est élevé, plus l'amélioration de ce score sera facile à obtenir. II est donc essentiel de ne plus travailler uniquement en pourcentage d'amélioration de score, mais aussi en évolution des scores globaux.

L'amélioration de $75 \%$ d'un score sous traitement (PASI 75) signifiet-elle une amélioration parallèle de $75 \%$ de la maladie? À partir de quelle diminution de la surface atteinte le patient commence-t-il vraiment à être soulagé ? Aucune étude ne cherche à répondre à cette question. II en est résulté un remplacement du PASI 90 par le PASI 75, sans autre justification qu'un effort de marketing pour montrer des résultats thérapeutiques plus encourageants. Plus fondamentalement, 
il faut clairement faire la distinction entre l'essai thérapeutique, qui étudie une large population, et la démarche thérapeutique, où le problème est de soulager un individu résolument unique et différent, et où c'est la satisfaction de chaque patient et non l'évolution d'un score qui définit le succès thérapeutique. Si l'on étudie une large population, il y a un parfait parallélisme entre l'importance de la surface corporelle atteinte par le psoriasis et l'altération de la qualité de la vie. Pour un patient individuel, cette concordance n'existe pas.

Une difficulté plus importante réside dans la comparaison de traitements nouveaux avec les traitements dits de référence. La comparaison de la cinétique d'évolution des scores sous deux traitements différents est particulièrement difficile à interpréter : le psoriasis est en effet une maladie chronique, alors que les traitements sont souvent comparés sur de courtes périodes de temps, de l'ordre de trois à six mois. Un traitement qui permettrait une amélioration progressive des lésions avec une quasi-disparition en un an pourrait être beaucoup plus utile qu'un traitement entraînant une disparition très rapide des lésions, suivie par un échappement, voire par un rebond. En fait, l'un et l'autre de ces traitements seront sans doute utiles, le premier comme traitement de fond, si sa tolérance le permet, le second pour faire face à une situation de crise. La comparaison de deux traitements permet donc beaucoup plus d'anticiper leurs modes d'utilisation respectifs que de considérer que l'un est supérieur à un autre. Dans le traitement des maladies chroniques, nous avons besoin de toutes les cinétiques d'activité. Les études contre médicament dit de référence sont donc absurdes.

Plus fondamentalement encore, il est clair aujourd'hui, pour tous les experts, que la stratégie de traitement du psoriasis, comme celle de beaucoup de maladies chroniques, ne repose pas sur un arbre décisionnel allant de traitements légers vers des traitements de plus en plus lourds et coûteux : ce type d'approche ne tient compte, en effet, que des médicaments, et fait abstraction du patient. La stratégie de traitement du psoriasis repose sur une approche individuelle, adaptée à chaque patient, éclairée par une évaluation globale réalisée comme décrite précédemment, et qui prend donc en compte les contraintes médicales, sociales, psychologiques et économiques propres à chaque patient. Dans ce contexte, il n'est pas rare de commencer par un traitement qui serait placé en troisième ligne dans un arbre décisionnel ne tenant compte que des médicaments.

La réponse aux traitements est trop souvent étudiée uniquement sur de larges populations. L'étude de souspopulations de patients éventuellement répondeurs est très importante. Un médicament soulageant très efficacement $10 \%$ des malades est un réel progrès thérapeutique, alors qu'un médicament soulageant médiocrement l'ensemble d'une population est sans doute moins utile. En fait, il faudra, dans le premier cas, essayer de trouver les tests permettant de repérer les répondeurs, tandis c'est la recherche de traitements associés, destinés à potentialiser une efficacité insuffisante, qui retiendra l'attention dans le second cas.

Une question parallèle, essentiellement éthique, est posée par le grand nombre de publications thérapeutiques existant actuellement sur le psoriasis. Est-il encore éthique de réaliser des études contre placebo dans le psoriasis? La sélection des nombreuses études thérapeutiques rigoureuses comportant un groupe placebo devrait permettre de mesurer l'effet placebo dans le psoriasis, permettant de définir, pour les études ultérieures, un seuil d'amélioration au-delà duquel on est certain qu'il s'agit d'un effet thérapeutique spécifique.

Lorsqu'un médicament est mis sur le marché, on connaît son efficacité et sa tolérance seulement à court terme, jamais à long terme. On ne connaît pas plus le service qu'il rendra vraiment, ni la place qu'il prendra dans la stratégie thérapeutique : on ne connaît pas son utilité. La ciclosporine, par exemple, a mis 12 ans après les premières utilisations dans le psoriasis comme traitement de «la dernière chance» pour trouver sa vraie place comme médicament systémique antipsoriasique modèle de première intention pour les jeunes femmes minces et hypotendues, en cures courtes. II est donc vital, pour la poursuite du progrès thérapeutique, d'autoriser la mise sur le marché d'un médicament dès que l'on connaît son efficacité et sa tolérance à court terme. Cette autorisation doit être assortie d'une liste de questions auxquelles l'industrie pharmaceutique devra répondre dans un laps de temps de 5 ans : ces questions ont pour but l'évaluation du service réellement rendu et la tolérance en conditions normales d'utilisation. Ce réexamen doit pouvoir aboutir à un retrait du médicament, ou à une nouvelle négociation du prix, ou même, si le service rendu n'est important que pour un trop petit nombre de malades pour que sa mise à disposition soit rentable, au passage de ce médicament dans la classe des médicaments orphelins. Ces règles existent, mais ne sont pas appliquées en Europe, et nombre de médicaments sont bloqués au départ sur des arguments inadaptés.

\section{Une nouvelle famille de médicaments}

Le psoriasis tue rarement. Cependant, on l'a compris, il retentit souvent gravement sur la qualité de la vie et crée souvent un réel handicap dans la relation du patient avec lui-même et avec les autres. Dans ce contexte, étant donné la gravité des souffrances psychologiques mises en jeu, il serait inacceptable que les patients souffrant de psoriasis ne puissent bénéficier des mêmes traitements que les patients souffrant d'autres maladies inflammatoires chroniques comme la polyarthrite rhumatoïde ou la maladie de Crohn.

Dans deux larges enquêtes, l'une américaine et l'autre européenne, une grande majorité de patients reprochent à leur médecin de ne pas leur proposer suffisamment les traitements systémiques, plus efficaces et plus commodes que les traitements locaux, même s'ils 
comportent une surveillance contraignante et des effets secondaires. Les traitements généraux actuellement disponibles pour traiter le psoriasis sont efficaces, mais jamais pour tous les patients: il existe des phénomènes d'intolérance et des contre-indications liées aux comorbidités. De plus, ils ne sont pratiquement jamais utilisables pendant toute la vie d'un patient, en raison d'effets toxiques cumulatifs. Enfin, pour un nombre non négligeable de patients, l'efficacité de chacun de ces traitements diminue avec le temps, obligeant à une stratégie de traitements alternatifs qui n'est pas toujours efficace.

Les traitements anti-inflammatoires et immunomodulateurs obtenus par les techniques de génie génétique soulèvent un espoir considérable. Les raisons en sont nombreuses : leurs cibles d'action sont très spécifiques, les uns inhibant l'activation lymphocytaire, les autres des cytokines pro-inflammatoires comme le TNF (tumor necrosis factor), cette spécificité laissant espérer une absence de toxicité d'organe; leurs mécanismes d'action sont très différents des molécules déjà existantes. De ce fait, ils peuvent résoudre de nombreuses situations d'échec thérapeutique; ils ont des profils pharmacodynamiques différents, ce qui permet de les adapter, de façon personnalisée, à la demande de chaque patient. Nous avons en effet besoin tout à la fois de traitements agissant rapidement, et de traitements agissant très progressivement, de traitements utilisables en traitement d'attaque, et de traitements principalement destinés à éviter les rechutes, de traitements efficaces sur la peau, et de traitements également efficaces sur le rhumatisme psoriasique. Enfin, l'absence de toxicité d'organe de ces nouveaux médicaments laisse espérer qu'ils pourront être associés sans difficulté aux autres traitements locaux ou systémiques. Il est clair, bien sûr, que le véritable service rendu par chacun de ces nouveaux médicaments ne sera connu que plusieurs années après leur mise à la disposition des patients. De même, on ne peut éliminer la possibilité d'effets toxiques apparaissant après plusieurs années d'utilisation, puisque chacun de ces médicaments modifie le profil d'activation lymphocytaire ou l'expression des cytokines dans l'organisme humain: la mise en place d'un suivi de cohortes de patients traités au long cours est donc une nécessité.

Les médicaments issus des biotechnologies remettent en cause une habitude réglementaire: aujourd'hui, l'autorisation de mise sur le marché n'est pas donnée à un médicament, mais à un médicament dans une maladie. Or, cette attitude est sans doute le plus grand frein au progrès thérapeutique : beaucoup de nouvelles molécules, et c'est en particulier le cas des molécules anti-inflammatoires d'origine biologique et des immunosuppresseurs, ont pour cible des mécanismes communs à de nombreuses maladies. Le coût des essais thérapeutiques est tel que les études ne sont faites que pour une ou un petit nombre de maladies, et le médecin ne peut utiliser le nouveau médicament pour traiter les autres indications qu'en étant hors la loi. Plus grave encore, beaucoup de molécules utiles sont abandonnées parce qu'elles ne concernent qu'un trop petit nombre de patients dans une maladie, alors que leur développement serait rentable si elles pouvaient être prescrite à tous les patients qui en ont besoin. II apparaît donc urgent d'autoriser la mise sur le marché d'un médicament pour son activité biologique dans toutes les maladies où cette activité peut être utile. II est plus urgent, encore, d'entamer une réflexion, au niveau international, entre les agences du médicament et les industries pharmaceutiques, sur les moyens de diminuer le coût de développement des médicaments, car une quantité d'études toxicologiques et cliniques sont totalement inutiles: les procédures administratives devraient être au service du progrès thérapeutique, et non son frein principal.

\section{Conclusions et perspectives: la solidarité, jusqu'où?}

L'évaluation de ces nouvelles molécules pose avec acuité une question éthique. Du fait de leur coût élevé, il est tentant de passer de l'évaluation du rapport efficacité/toxicité à celui du rapport efficacité/toxicité + coût. Si les règlements de I'Union européenne indiquent clairement que le coût d'un médicament ne doit pas intervenir dans sa mise à la disposition des citoyens, il est évident que le coût important de ces molécules est, en pratique, un handicap majeur pour leur développement dans le traitement de maladies qui ne mettent pas la vie en danger. La question se pose donc de savoir jusqu'où ira la solidarité dans la prise en charge du handicap peau, et qui décidera du niveau de cette solidarité. $\diamond$

\section{SUMMARY}

Psoriasis: evolution and revolution

Psoriasis is a model disease in dermatology. It is a common disease that affects at least 2 to $3 \%$ of the population. It is an illness characterized by an excessive reaction of the skin, in term of proinflammatory cytokines release, to no specific attacks: these attacks can be immunological, mechanical, metabolic, drug-induced or psychological. This excessive reaction is characterized by epidermal proliferation combined with incomplete terminal differentiation, as well as an inflammatory response responsible for the chronic nature of the lesions. The way to understand psoriasis is therefore to reach a better appreciation of the messages that enable the skin cells to initiate an inflammatory response, and by better understanding the way in which the inflammatory cells responsible for innate and acquired immune responses are capable of bringing about proliferation and abnormal epidermal differentiation. Taking an interest in psoriasis is therefore taking an interest in all facets of skin physiology and in all the ways the skin reacts to attacks from the environment. Every year for more than thirty years, more than 300 publications have endeavoured to explore one aspect or another of psoriasis from a clinical, epidemiological, physiopathological or therapeutic point of view. There is no new technique for observing the skin that has not been immediately applied to the study of psoriasis - which is privileged to enjoy the reflected progress made in dermatology. Nor has 
psoriasis remained untouched by whims of fashion, all manner of scenarios having been suggested to explain it, right from a scarring disease to an autoimmune illness through a genetic or psychosomatic disorder. Psoriasis is at the origin of a medical revolution mounted to supplement and enhance the effectiveness of evidence-based medicine; it is the "patient-centred medicine". Psoriasis only exceptionally jeopardizes life. Conversely, it is a disease that does affect quality of life. The patient alone must be the judge of his or her quality of life, and it is therefore up to the patient, not the doctor, to gauge the severity of psoriasis and hence decide on reasonable therapeutic indications. Psoriasis, then, cannot be treated without placing the patient, not the illness, at the centre of therapeutic negotiations. The 20th century has seen the disease targeted by boundless efforts; the 2lst century will see the development of medical techniques that allow the patient, in all its complexity, to be positioned at the centre of therapeutic efforts. This revolution began in dermatology, centring around psoriasis, and is spreading progressively to all chronic disorders and all disciplines. New quite interesting therapeutic weapons are available from a few months making possible to better adjust the therapeutic strategies of psoriasis to the patients needs but they are expensive opening again the debates on the limit of the social solidarity. $\diamond$

\section{RÉFÉRENCES}

1. Krueger GG, Chambers DA, Shelby J. Involved and uninvolved skin from psoriatic subjects: are they equally diseased? Assessment by skin transplanted to congenitally athymic (nude) mice. J Clin Invest 1981 ; 68 : 1548-57.

2. Fraki JE, Briggaman RA, Lazarus GS. Transplantation of psoriatic skin onto nude mice. J Invest Dermatol $1983 ; 80: 31 \mathrm{~s}-35 \mathrm{~s}$

3. Dzionek A, Sohma Y, Nagafune J, et al. BDCA-2, a novel plasmacytoid dendritic cell-specific type II C-type lectin, mediates antigen capture and is a potent inhibitor of interferon alpha/ beta induction. J Exp Med 2001 ; 194 : 1823-34.

4. Saiag P, Coulomb B, Lebreton C, et al. Psoriatic fibroblasts induce hyperproliferation of normal keratinocytes in a skin equivalent model in vitro. Science $1985 ; 230: 669-72$.

5. Wrone-Smith T, Nickoloff BJ. Dermal injection of immunocytes induces psoriasis. J Clin Invest $1996 ; 98: 1878-87$.

6. Michel $L$, Sextius $P$, Jeanlouis $F$, et al. Interaction between $T$ cells and fibroblasts in psoriasis. Exp Dermatol 2006 (soumis pour publication).

7. Sano S, Chan KS, Carbajal S, et al. Stat3 links activated keratinocytes and immunocytes required for development of psoriasis in a novel transgenic mouse model. Nat Med 2005 ; $11: 43-9$.

8. Rainer Zenz, Robert Eferl, Lukas Kenner, et al. Psoriasis-like skin disease and arthritis caused by inducible epidermal deletion of Jun proteins. Nature 2005; $437: 369-75$

9. Mazer JM, Fayard V, Dubertret L. Treatment of psoriasis by pulsed dye laser : short term and long term (3 years) results. Arch Dermatol 2006 (soumis pour publication).

10. Dubertret L, Bertaux B, Fosse M, Touraine R. Psoriasis : a defect in the regulation of epidermal proteases, as shown by serial biopsies after cantharidin application. Br J Dermatol 1984 ; $110: 405-10$.

11. Dubertret L, Chastang C, Beylot C, et al. Maintenance treatment of psoriasis by Tigason : a double-blind randomized clinical trial. Br J Dermatol 1985; $113: 323-30$.
TIRÉS À PART

L. Dubertret

\section{UN OUVRAGE INDISPENSABLE : À L'ÉTUDIANT POUR ÉTUDIER, RÉVISER ET RÉUSSIR L'ÉPREUVE DE BIOPHYSIQUE DU PCEM, ET Ȧ L'ENSEIGNANT COMME SUPPORT DE COURS}

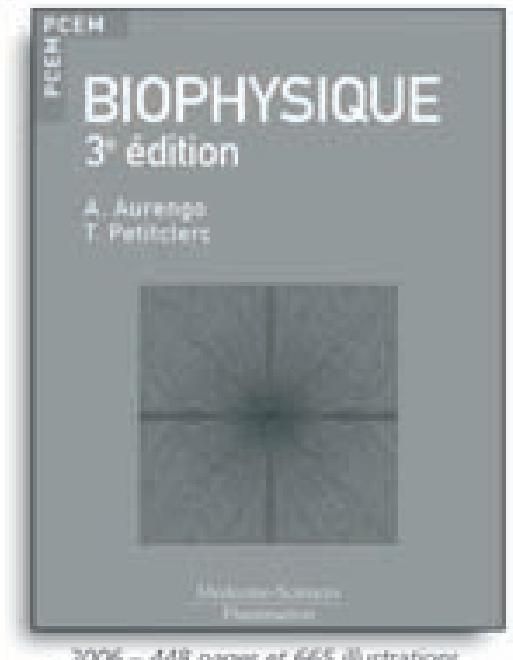

BIOPHYSIQUE - 3’édition André AURENGO et Thierry PETITCLERC, professeurs de biophysique ou CHU Pitié-Salpétrière.

Clait, exhaustif, cet ouvrage rassemble en un seul volume, lensemble des éléments de biophysique ayant une application medicale. Résolument pédagogique, it expose, de façon explicite et accessible, des notions utiles a la comprehension de la physiologie, de la physiopathologie et des explorations fonctionnelles. If comporte 28 chapitres qui traitent de tous les items du programme de PCEM 1 ; depuis l'équilore acido-busique, l'equilbre hydrosode, rélectrophysiologie cellulaire, l'activité electrique du coeur jusqu'a l'IRM, l'echographie, la scintigraphie, les applications biologiques des radioblements, en passant par l'étude de la radioactivite, des effets biologiques des rayonnements ionisants, etc:

Très richement illustré de 665 schémas, tableaux et documents d'examen, cet ouvrage unique propose de plus, 115 exercices et 150 QCM avec leurs corriges détaillés en fin d'ouvrage.

$2006=449$ pages et 665 illistrations

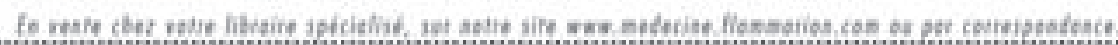
BON DE COMMANDE a reloutner a

FLAMMARION MHDFCINE - 87. Quai Panhard et Levassor - 75647 PARIS Cedex 13

NOM Fienom Whe:

Cocle posial Adrese

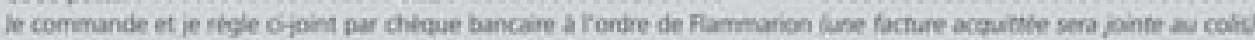
I BiOPHYSIQUE A. Aurengo et T. Pettclerc ( + SE de participation aux frais de port) soit 